\title{
Comparative evaluation of the traffic flow volatility forecasting models
}

\author{
Jia-Wei Lu ${ }^{1, a}$, Hong-Jun Xue ${ }^{2, b}$, Guang-Jiao Chen ${ }^{2, c}$, You Zhou ${ }^{1, \mathrm{~d}}$ and Jing-Xin Xia ${ }^{1, \mathrm{e}}$ \\ ${ }^{1}$ Intelligent Transportation System Research Center, Southeast University, \\ Nanjing 210096, China \\ ${ }^{2}$ Nantong Road Management Department, \\ Nantong 226001, China \\ E-mail:"alujiaweiseu@163.com, ${ }^{b} 23536417 @ q q . c o m,{ }^{c} 2524892122 @ q q . c o m$, \\ d120880558@qq.com, ${ }^{\mathrm{e}}$ jingxinxia@yahoo.com.cn \\ www.seu.edu.cn
}

\begin{abstract}
Accurate and reliable short-term traffic flow forecasting is essential for advanced traveler information systems and proactive traffic signal control systems. However, the majority of current studies mainly concentrate on short-term traffic flow level forecasting. To improve the forecasting reliability of traffic flow parameters, short-term traffic flow uncertainty forecasting has been emphasized and studied gradually, and many models had been developed. Among these models, generalized autoregressive conditional heteroscedasticity (GARCH) and stochastic volatility (SV) model is considered to have high accuracy and reliability. Based on traffic flow data collected from urban roads, forecasting methods of forecasting performance of short-term traffic flow uncertainty are studied and compared under different roads and traffic conditions in this paper. The results show that the performance of the ARIMA-GARCH, VAR-MGARCH, and ARIMA-SV models for short-term speed forecasting is better than the performance for volume forecasting. Moreover, the accuracy performance of VAR-MGARCH model is better than the ARIMA-SV and ARIMA-GARCH models, but the ARIMA-SV model performs better than the ARIMA-GARCH and VAR-MGARCH models in terms of the short-term uncertainty forecasting.
\end{abstract}

Keywords: Traffic Flow Volatility; Short-term Forecasting; Performance Evaluation Comparative Analysis.

\section{Introduction}

Advanced Traveler Information Systems (ATIS) and Active Signal Control Systems (ASCS) play a central role in Intelligent Transportation Systems (ITS). By applying the two advanced traffic systems, transportation system efficiency has been improved and traffic congestions have been released in a certain extent. As an essential and fundamental technology of ATIS and the ASCS, short-term traffic flow forecasting has been studied extensively in recent decades. 
Short-term traffic flow forecasting starts from the forecasting of univariate traffic flow parameter, including traffic volume, traffic speed, travel times, and etc. Tremendous univariate prediction models have been proposed including parameter models such as time series models [1-3], Kalman filtering [4-5], support vector machine [6-7], and some non-parameter models such as nonparametric regressive model[8-9] and neural network model[10-12]. To further improve the short-term forecasting accuracy, some multivariate models was introduced to calibrate the relationships between different traffic flow variables at a traffic station or the same variable at different traffic stations. The most popular multivariate forecasting models include state space model[13], multivariable nonparametric regression model[14] and vector auto-regression model[15-16]. Whatever univariate or multivariate, the above models focus on improving the forecasting accuracy through point estimation techniques.

Recently, the uncertainty problem in traffic forecasting has attracted much attention, since forecasting reliability is also an important measure in traffic flow forecasting. Therefore, some variance-based models based on time series theory have been proposed to capture and predict the volatility of traffic flow variables[17]. In details, the volatility or uncertainty of traffic flow variables is quantified by the standard deviations or variances through interval estimation techniques. These variance-based models can be classified broadly into two categories, i.e. the conditional variance models[18] and the stochastic volatility (SV) models[19]. Kamarianakis et al.[20] applied a standard generalized autoregressive conditional heteroscedasticity (GARCH) model to verify the volatility of relative speed. The GARCH model was also used by Guo and Williams[21] to capture and predict traffic speed volatility. Considering the inherent relationship between traffic volume and speed, Xia et al.[22] constructed a multivariate GARCH model for calibrating and forecasting of traffic volume and speed volatility. Tsekeris and Stathopoulos[23] used a stochastic volatility model to forecast the volatility of traffic speed through measured traffic speed and occupancy data.

Although the two types of variance-based models were verified suitable in short-term traffic flow volatility forecasting, none has been told to produce better performance. External factors such as traffic characteristic at different road types or traffic congestion levels at different time periods may affect the forecasting performance significantly. In this research, the accuracy and reliability performance for these the two types of volatility model is evaluated and compared in details. The results of the performance evaluation and comparison can provide practical advices for model selection under different circumstances. 


\section{The Short-term Forecasting Models}

Two types of volatility models, i.e. the GARCH and SV models based on time series theory are studied in this paper. For the GARCH-type models, both univariate and multivariate GARCH model are discussed. Because autocorrelation usually exists in traffic flow series, it is necessary to build a mean forecasting model first to eliminate the autocorrelation. Therefore, the ARIMA and VAR models are firstly proposed for forecasting traffic flow levels. Second, the GARCH, MGARCH and SV models are proposed for capturing the heteroscedasticity in traffic flow series. To compare volatility forecasting performance of GARCH and SV model, ARIMA-GARCH, VAR-MGARCH and ARIMA-SV model are constructed. In these three models, ARIMA and VAR model are built to forecast traffic flow level, while GARCH and SV model capture heteroscedasticity of the residuals generated by the mean forecasting models.

\subsection{Mean forecasting model}

\subsubsection{The univariate ARIMA model}

$\operatorname{ARIMA}(p, d, q)$ is a commonly used time series model for the mean value forecasting of traffic parameters. In this model, $p$ is the autoregressive order, $q$ is the moving average order, $d$ is the differential order. The general form of $\operatorname{ARIMA}(p, d, q)$ model can be defined as:

$$
\phi_{p}(B)(1-B)^{d} y_{t}=\theta_{0}+\theta_{q}(B) u_{t}
$$

Where $y_{t}$ is original data series, and it refers to traffic flow parameters in this paper; $(1-B)^{d} y_{t}$ is the $d$-order differential series of the original data series $y_{t}$ to meet the prerequisite of ARIMA model; $u_{t}$ is the white noise process whose mean value is 0 and variance is a constant $\sigma_{u}{ }^{2}$; $\theta_{q}(B)=1-\theta_{1} B-\cdots-\theta_{q} B^{q} \quad$ is the autoregressive operator and $\theta_{q}(B)=1-\theta_{1} B-\cdots-\theta_{q} B^{q}$ is the moving average operator. Because the original traffic flow is usually stationary after first order differenced, the value of $d$ is always taken as $1[24]$.

\subsubsection{The multivariate VAR model}

Due to the stable relationship between traffic flow parameters, the VAR model that could take care the relationship is usually used to forecast the means of traffic flow parameters. The general form of the VAR model is defined as: 


$$
\boldsymbol{Y}_{t}=\phi_{0}+\phi_{1} \boldsymbol{Y}_{t-1}+\boldsymbol{\phi}_{2} \boldsymbol{Y}_{t-2}+\cdots+\boldsymbol{\phi}_{p} \boldsymbol{Y}_{t-p}+\boldsymbol{U}_{t}
$$

Where $\boldsymbol{Y}_{t}=\left[y_{1}, \cdots y_{N}\right]^{T}$ is a variable matrix in $N$ dimensions at the moment of $t ; N$ is the number of variables; $\phi_{0}$ is a constant vector; $\phi_{1}, \phi_{2}, \ldots, \phi_{p}$ is a parameter matrix in $N \times N$ dimensions; $p$ is the autoregressive order and $\boldsymbol{U}_{t}$ is a stochastic error term.

Similar to the univariate time series model, the VAR model requires each variable series to be stable. Therefore, the original traffic flow series can be differenced in first order as the ARIMA model. In this sense, Eq.(2) can be transformed into the following form as:

$$
\Delta \boldsymbol{Y}_{t}=\prod \boldsymbol{Y}_{t-1}+\sum_{i=1}^{p-1} \Gamma_{i} \Delta \boldsymbol{Y}_{t-i}+\boldsymbol{U}_{t}
$$

Where $\Delta \boldsymbol{Y}_{t}$ is the series after first order difference; $\boldsymbol{\Gamma}_{i}=\sum_{j=i+1}^{p} \boldsymbol{\phi}_{j}(i=1,2, \ldots, p-1)$ and $\boldsymbol{\Pi}=\sum_{i=1}^{p} \boldsymbol{\phi}_{i}-\boldsymbol{I}$, in which $\boldsymbol{I}$ is the identity matrix $^{[18]}$.

\subsection{Volatility forecasting model}

\subsubsection{The univariate GARCH model}

The GARCH $(p, q)$ model can be used to describe and calculate the conditional heteroscedasticity when it exists in time series. The main idea is that the conditional variance of the perturbation term depends on the square errors of the previous period. The general form of the $\operatorname{GARCH}(p, q)$ model can be defined as:

$$
\begin{gathered}
u_{t}=\sigma_{t} e_{t} \\
\sigma_{t}^{2}=\alpha_{0}+\sum_{i=1}^{p} \alpha_{i} u_{t-i}{ }^{2}+\sum_{i=1}^{q} \beta_{i} \sigma_{t-i}{ }^{2}
\end{gathered}
$$

Where $\alpha_{i}$ and $\beta_{i}$ are the non-negative constants; $u_{t}$ is the residual at time $t$ generated by a mean forecasting model, $e_{t} \sim \operatorname{IIN}(0,1)$ and $\sigma_{t}^{2}$ is the conditional variance at time $t$. As for time series containing heteroscedasticity, $\operatorname{GARCH}(1,1)$ model is adopted to remove heteroscedasticity [19], so $p$ and $q$ are taken as 1 respectively in this paper.

\subsubsection{The multivariate GARCH model}

Similar to the VAR model, the univariate GARCH model is extended to a multivariate model, namely Multivariate GARCH (MGARCH) model to 
improve the uncertainty forecasting performance for the traffic flow variables. The basic structure of a $\operatorname{MGARCH}(p, q)$ is defined as:

$$
\boldsymbol{U}_{t}=\sqrt{\boldsymbol{H}_{t}} \boldsymbol{e}_{t}
$$

$$
\operatorname{Vech}\left(\boldsymbol{H}_{t}\right)=\boldsymbol{W}+\sum_{i=1}^{q} \boldsymbol{A}_{i} \operatorname{Vech}\left(\boldsymbol{U}_{t-i} \boldsymbol{U}_{t-i}^{T}\right)+\sum_{j=1}^{p} \boldsymbol{B}_{j} \operatorname{Vech}\left(\boldsymbol{H}_{t-j}\right)
$$

Where $\boldsymbol{A}_{i}, \boldsymbol{B}_{j}$ are both coefficient matrices; $\boldsymbol{U}_{t} \mid \boldsymbol{\Omega}_{-1} \sim \boldsymbol{N}\left(0, \boldsymbol{H}_{t}\right)$, $\boldsymbol{e}_{t} \sim \operatorname{IIN}(0,1), \boldsymbol{\Omega}_{-1}$ is the data set at time $t-1 ; \boldsymbol{H}_{t}$ is a $N \times N$-dimensional positive definite matrix, and it is also computable using $\boldsymbol{\Omega}_{-1} ; \operatorname{Vech}(\bullet)$ is a vector operator and $\boldsymbol{W}$ is $\frac{N(N+1)}{2} \times 1$-dimensional constant vector.

The MGARCH model has two major difficulties in the process of building the model. First, the increase of variable number will lead to the 'Curse of dimensionality', resulting in a substantial increase in the complexity estimates of model parameters. Second, with the increase of number of variables, the difficulty to ensure the positive definiteness of the covariance matrix will also increase. Therefore, Engel and Kroner [25] proposed a new form of MGARCH, namely the BEKK model, which has fewer parameters to be estimated, and the positive definite covariance matrix is easier to be satisfied. In this paper, the BEKK model is chose to build the MGARCH model to capture the heteroscedasticity in traffic flow parameters series. Let:

$$
\boldsymbol{A}_{i}=\sum_{k=1}^{K}\left(\tilde{\boldsymbol{A}}_{i k} \otimes \tilde{\boldsymbol{A}}_{i k}\right)^{T}, \quad \boldsymbol{B}_{j}=\sum_{k=1}^{K}\left(\tilde{\boldsymbol{B}}_{j k} \otimes \tilde{\boldsymbol{B}}_{j k}\right)^{T}
$$

Where $\tilde{\boldsymbol{A}}_{i k}$ and $\tilde{\boldsymbol{B}}_{i k}$ are $N$-dimensional square matrices and $\otimes$ indicates matrix Kronecker product. Then, the $\operatorname{MGARCH}(q, p)$ model can be transformed as:

$$
\begin{aligned}
& \operatorname{Vech}\left(\boldsymbol{H}_{t}\right)=\boldsymbol{W}+\sum_{i=1}^{q} \sum_{k=1}^{K}\left(\tilde{\boldsymbol{A}}_{i k} \otimes \tilde{\boldsymbol{A}}_{i k}\right)^{T} \operatorname{Vech}\left(\boldsymbol{U}_{t-i} \boldsymbol{U}_{t-i}{ }^{T}\right) \\
& +\sum_{j=1}^{p} \sum_{k=1}^{K}\left(\tilde{\boldsymbol{B}}_{j k} \otimes \tilde{\boldsymbol{B}}_{j k}\right)^{T} \operatorname{Vech}\left(\boldsymbol{H}_{t-j}\right)
\end{aligned}
$$

Like univariate GARCH model, $p$ and $q$ in the model are taken as 1 too.

\subsubsection{The SV model}

The GARCH model describes the volatility characteristics of time series that are discrete and observable. The GARCH model calculates the volatility of the time 
series based on the past observations and square errors. Comparatively, the volatility of the time series in a SV model is determined by a non-observable stochastic process. In other words, the volatility captured by the GARCH model is parameterized as a function of past shocks only, but the SV model can take latent state variables into considerations. The latent state variables may be related to the interaction between vehicles or the effect of traffic management measures. Therefore, the SV model is considered suitable for forecasting traffic flow series with strong uncertainty. The basic discrete SV model is expressed as:

$$
\begin{gathered}
u_{t}=\sigma_{t} \varepsilon_{t} \\
\ln \sigma_{t}^{2}=\alpha+\beta\left(\ln \sigma_{t-1}^{2}-\alpha\right)+\eta_{t}
\end{gathered}
$$

Where $\alpha$ and $\beta$ are constants; $u_{t}$ represents the observed value after the removal of mean at time $t ;\left\{\varepsilon_{t}\right\}$ is a martingale difference series; Perturbation terms $\varepsilon_{t}$ and $\eta_{t}$ can be contemporaneous correlation, $\varepsilon_{t} \sim \operatorname{IIN}(0,1)$ and $\eta_{t} \sim \operatorname{IIN}\left(0, \sigma_{\eta}^{2}\right), \sigma_{\eta}$ is unknown.

\section{Model Validation}

\subsection{Data description}

Tab. 1 Basic information of traffic flow data

\begin{tabular}{ccccc}
\hline Road type & Road name & Station & Calibration dataset & Evaluation dataset \\
\hline Expressway & $\begin{array}{c}\text { Beijing-Xizang } \\
\text { expressway }\end{array}$ & 51008 & $2009 / 9 / 8$ & $2009 / 9 / 9 \sim 2009 / 9 / 15$ \\
& & 51004 & $2009 / 9 / 8$ & $2009 / 9 / 9 \sim 2009 / 9 / 15$ \\
Major arterial & Qianjin road & 108121 & $2015 / 6 / 15$ & $2015 / 6 / 16 \sim 2015 / 6 / 22$ \\
& & 108221 & $2015 / 6 / 15$ & $2015 / 6 / 16 \sim 2015 / 6 / 22$ \\
Minor arterial & Tongfeng road & 102001 & $2015 / 5 / 13$ & $2015 / 5 / 14 \sim 2015 / 6 / 20$ \\
& & 103001 & $2015 / 5 / 13$ & $2015 / 5 / 14 \sim 2015 / 6 / 20$ \\
\hline
\end{tabular}

In this paper, traffic flow data from Beijing and Kunshan were obtained, and three types of roads - expressway, major arterial, and minor arterial-were selected. The traffic volume and speed measurements from microwave vehicle detector stations installed along each road type were used. The volume and speed data were aggregated into 5 minutes and were divided into a calibration dataset and an evaluation dataset. The details of the data information are shown in Table 1. 


\subsection{Mean forecasting model construction}

The ARIMA and VAR models are used for forecasting the mean of traffic volume and speed, and they belong to time series models. The premise of the time series model is that the sequence of the traffic variables should be stable. To ensure the two models can be used, the ADF test was used to test the stationarity of traffic volume and speed in 5 minutes. Table 2 shows the results of ADF test, in which $t_{g}$ is the $t$-statistic value of the unit root. If $t_{g}$ is less than critical value of $\mathrm{ADF}$ at a significance level ( 0.05 in this paper), the series is stationary. As the results show, the original aggregated data series are nonstationary, but the new $t_{g}$ value are significantly less than the critical value for the first order differential series, indicating that the first order differential series is stationary.

Tab. 2 Stationarity test results based on augmented dickey-fuller(ADF) approach

\begin{tabular}{ccccc}
\hline Station & Traffic variables & $t_{g}$ & Critical value & Stationarity \\
\hline \multirow{2}{*}{51008} & volume & $-0.90 /-16.37$ & $-2.87 /-1.94$ & non-stationary/stationary \\
& speed & $-2.51 /-15.59$ & $-2.87 /-1.94$ & non-stationary/stationary \\
\multirow{2}{*}{1084} & volume & $-0.91 /-18.18$ & $-2.87 /-1.94$ & non-stationary/stationary \\
& speed & $-2.74 /-14.11$ & $-2.87 /-1.94$ & non-stationary/stationary \\
\multirow{2}{*}{108221} & volume & $-1.67 /-5.45$ & $-2.87 /-1.94$ & non-stationary/stationary \\
& speed & $-2.22 /-12.82$ & $-2.87 /-1.94$ & non-stationary/stationary \\
& volume & $-1.53 /-24.31$ & $-2.87 /-1.94$ & non-stationary/stationary \\
& speed & $-1.62 /-19.37$ & $-2.87 /-1.94$ & non-stationary/stationary \\
& volume & $-1.63 /-15.51$ & $-2.87 /-1.94$ & non-stationary/stationary \\
& speed & $-2.28 /-19.95$ & $-2.87 /-1.94$ & non-stationary/stationary \\
& volume & $-1.64 /-14.87$ & $-2.87 /-1.94$ & non-stationary/stationary \\
\hline Note: figures before & speed & $-2.09 /-14.72$ & $-2.87 /-1.94$ & non-stationary/stationary \\
\hline
\end{tabular}

In addition, reasonable lag order selection has a significant impact on the performance of the AIRIMA and VAR models. A large lag order might better reflect the dynamics of the traffic flow, but it also brings more parameters to be estimated, which limits the application of the models. Therefore, the BIC criterion was selected to identify lag order for the ARIMA and VAR models. Finally, the ARIMA $(0,1,1)$ and VAR (3) models are selected to forecast the mean of the traffic parameters.

After constructing the mean forecasting models, the correlation of the residual series was checked. If the mean forecasting model is adequate, then the residual series should behave as a white noise ${ }^{[26]}$. The test result shows that 
correlation does not exist in the residual series, indicating that the time series models selected in this paper are adequate for traffic flow mean forecasting.

\subsection{Volatility forecasting model construction}

Before constructing the volatility forecasting models, the heteroscedasticity test was conducted to verify whether a time-varying conditional variance exists in the residual sequence generated by the mean forecasting models. For the single variable model (ARIMA), ARCH LM test was used to determine the existence of ARCH effect. The original hypothesis of ARCH LM is that the residual sequence does not have ARCH effect, the alternative hypothesis is that there is $\mathrm{ARCH}$ effect.

When significant level of $\alpha$ and degrees of freedom $q$ are given, if $L M<\chi_{\alpha}^{2}(q)$, the original hypothesis $H_{0}$ will be accepted. That is, the residual series is not heteroscedastic. If $L M>\chi_{\alpha}{ }^{2}(q)$, the original hypothesis $H_{0}$ will be rejected. That is, the residual series is heteroscedastic. ARIMA $(0,1,1)$ is selected as level forecasting model for ARCH LM test in this paper, and the test results are shown in Table 3, which the $P$-value denotes the probability for not rejecting $H_{0}$. It is clear that original hypothesis $H_{0}$ cannot be accepted at the confidence level of $95 \%$.That is to say heteroscedasticity exists in traffic flow series for each station, and it is necessary to construct GARCH model and SV model to capture it.

As an example, table 3 also shows the heteroscedasticity test of ARIMA $(0,1,1)$-GARCH $(1,1)$ model. It is obvious that heteroscedasticity does not exist in the residual series, showing that the GARCH model can be used to the traffic flow volatility forecasting.

Tab. 3 Heteroscedasticity test results of $\operatorname{ARIMA}(0,1,1)$ model

\begin{tabular}{ccccc}
\hline \multirow{2}{*}{ Station } & $\mathbb{N R}^{2}$ & $P$-Value & $\mathbb{N R}^{2}$ & speed \\
\cline { 2 - 5 } 51008 & $20.35 / 0.32$ & $0.00 / 0.96$ & $12.47 / 1.25$ & $0.01 / 0.74$ \\
51004 & $26.08 / 2.07$ & $0.00 / 0.56$ & $18.03 / 1.80$ & $0.00 / 0.61$ \\
108121 & $9.50 / 1.41$ & $0.02 / 0.70$ & $9.48 / 1.94$ & $0.02 / 0.58$ \\
108221 & $32.20 / 0.31$ & $0.00 / 0.96$ & $8.48 / 0.53$ & $0.04 / 0.91$ \\
102001 & $13.06 / 0.64$ & $0.00 / 0.89$ & $16.19 / 0.72$ & $0.00 / 0.87$ \\
103001 & $20.39 / 0.36$ & $0.00 / 0.95$ & $17.17 / 1.84$ & $0.00 / 0.60$ \\
\hline
\end{tabular}

Note: figures before'/' refer to test results of $\operatorname{ARIMA}(0,1,1)$, figures after'/' refer to test results of ARIMA(0,1,1)-GARCH(1,1) 


\section{Performance Evaluation}

In this section, the forecasting performance of the proposed models is evaluated under different road and traffic conditions. Three measures, including mean absolute percentage error (MAPE), the width of confidence interval (CI), and kickoff percentage (KP), are chosen to evaluate the accuracy and reliability of the models. The CI is calculated using variance predicted by the conditional heteroscedasticity model under the confidence level of $95 \%$, and the kickoff percentage is calculated from the observed values that fall outside the confidence interval accounted for the proportion of the overall samples.

\subsection{Performance evaluation under different road type}

Tab. 4 Performance comparison under different road type

\begin{tabular}{|c|c|c|c|c|c|c|c|}
\hline \multirow[b]{2}{*}{ Station } & \multirow[b]{2}{*}{ Model } & \multicolumn{3}{|c|}{ Volume } & \multicolumn{3}{|c|}{ Speed } \\
\hline & & MAPE & $\mathrm{CI}$ & $\mathrm{KP}$ & MAPE & $\mathrm{CI}$ & KP \\
\hline \multirow[t]{3}{*}{51008} & ARIMA-GARCH & 8.48 & 81.52 & 6.50 & 7.47 & 20.34 & 5.01 \\
\hline & VAR-MGARCH & 8.75 & 80.79 & 6.25 & 7.48 & 20.30 & 4.81 \\
\hline & ARIMA-SV & 8.50 & 74.90 & 6.94 & 7.42 & 18.27 & 5.65 \\
\hline \multirow[t]{3}{*}{51004} & ARIMA-GARCH & 10.52 & 77.00 & 5.46 & 3.90 & 13.64 & 4.22 \\
\hline & VAR-MGARCH & 10.64 & 76.42 & 5.16 & 4.00 & 13.46 & 5.16 \\
\hline & ARIMA-SV & 10.46 & 71.78 & 5.46 & 3.90 & 12.93 & 4.91 \\
\hline \multirow[t]{3}{*}{108121} & ARIMA-GARCH & 11.20 & 21.38 & 5.06 & 7.52 & 9.33 & 5.06 \\
\hline & VAR-MGARCH & 12.56 & 21.44 & 8.23 & 7.50 & 30.95 & 0.05 \\
\hline & ARIMA-SV & 11.27 & 20.12 & 4.32 & 7.45 & 8.82 & 4.21 \\
\hline \multirow[t]{3}{*}{108221} & ARIMA-GARCH & 12.91 & 18.16 & 5.51 & 5.25 & 9.42 & 4.86 \\
\hline & VAR-MGARCH & 12.83 & 14.63 & 9.03 & 5.19 & 10.22 & 3.42 \\
\hline & ARIMA-SV & 13.15 & 17.28 & 3.13 & 5.25 & 8.68 & 4.66 \\
\hline \multirow[t]{3}{*}{102001} & ARIMA-GARCH & 14.79 & 27.95 & 1.79 & 8.34 & 23.02 & 1.69 \\
\hline & VAR-MGARCH & 14.20 & 22.05 & 3.22 & 8.23 & 20.34 & 2.03 \\
\hline & ARIMA-SV & 14.58 & 17.47 & 5.11 & 8.34 & 15.76 & 5.90 \\
\hline \multirow[t]{3}{*}{103001} & ARIMA-GARCH & 17.85 & 32.88 & 4.22 & 10.89 & 15.07 & 6.94 \\
\hline & VAR-MGARCH & $\mathbf{1 7 . 8 5}$ & 31.96 & 4.37 & 10.86 & 16.22 & 4.37 \\
\hline & ARIMA-SV & 17.87 & 28.90 & 5.46 & 10.90 & 14.90 & 5.75 \\
\hline
\end{tabular}

Traffic characteristics on expressway, major arterial, and minor arterial roads differ from each other. In general, the traffic flow on expressway is continuous with large traffic volume and high speed. By contrast, the major arterial roads are significantly influenced by the intersection along the road, so the traffic volume and speed will decrease. Similarly, the minor arterials usually have low 
speed, low flow rate. The performance of the proposed models under different road types is given in Table 4. Overall, the accuracy of the model for speed significantly higher than the volume prediction, but the accuracy of the three models to forecast the volume or speed has no obvious difference at the same station. Moreover, the ARIMA - SV model is better than the ARIMA-GARCH and VAR-MGARCH models for the volatility forecasting.

In terms of accuracy, the performance of each model for volume and speed forecasting decreases with the decline of the road grade. For volume forecasting, the MAPE values for expressway are about $9 \%$, falling to about $12 \%$ for the major arterial, and then to $16 \%$ for the minor arterial. For the speed forecasting, the MAPE values for the expressway, major arterial, and minor arterial are about $5 \%, 6 \%$ and $9 \%$, respectively. By comparing the forecasting results for both the volume and speed, it is found that the forecasting accuracy of the three models for speed forecasting is obviously better than that for the volume forecasting on all the three types of road. Furthermore, the results for volume forecasting are found sensitive to the change of road types, leading to the accuracy gap between volume and speed become larger with the decrease of the road grade. The decline of forecasting accuracy on the arterials is supposed to be related to the possible traffic interference from the intersections, non-motor vehicles and pedestrians. In addition, although no significant difference of the forecasting accuracy exists among the three models, the VAR-MGARCH model by considering the relationship between volume and speed shows a narrow margin on the major arterial and minor arterial roads for both volume and speed forecasting (see the values that are marked in bold when VAR-MGARCH model gets smallest MAPE value).

In terms of volatility, table 4 shows that the average of confident interval decreases with the decrease of road grade. The reliability of the forecasting on the minor arterial is slightly lower than major arterial. The underlying reason is that the traffic volume or speed on the major arterial is similar to those on the minor arterial road in this paper. In this sense, the volume and speed on the minor arterial road present a strong er variability when compared to the major arterial. When comparing the GARCH-type and SV model performance, it is observed that the ARIMA-SV model for both volume and speed forecasting is better than the ARIMA-GARCH and VAR-GARCH models on the same station, in terms of the average width of the confidence interval. Specifically, ARIMA$\mathrm{SV}$ model produce the smallest average confidence interval while no significant difference of the KP values among the three models. 


\subsection{Performance evaluation under different traffic conditions}

The speed is usually used to represent the traffic conditions for urban roads. Therefore, the performance of the three models was evaluated under different speed intervals that were simply divided by different road types. If the sample size is less than 10 in one interval, the performance evaluation will not be conducted to ensure the stability of the evaluation results. The performance results of the three models are shown in table 5 and table 6 , in which '-' means no enough sample in this interval.

In terms of accuracy, obvious difference of forecasting accuracy exists among the different speed intervals on all the types of road. Judged by the value of MAPE, as for the expressway and major arterial, the forecasting result is stable and accurate when the speed ranges from $50 \sim 70 \mathrm{~km} / \mathrm{h}$, and the decrease of accuracy is found when the speed is lower than $50 \mathrm{~km} / \mathrm{h}$ or higher than $70 \mathrm{~km} / \mathrm{h}$. As for the minor arterial, the forecasting result is stable and accurate when speed ranges from $30 \sim 50 \mathrm{~km} / \mathrm{h}$, and the decrease of accuracy is found when the speed is out of that interval. The results fit the fact that traffic state is non-stable when average speed is low (congestion) or high (free flow). In addition, there is no significant difference in the forecasting accuracy among the three models at the same station and the same speed interval. The bold figures in the table refer to the minimum values calculated at the same station and the same speed interval. On the whole, the accuracy of the VAR-MGARCH model is a little better than that of the ARIMA-GARCH and GARCH-SV models. Specifically, the performance of the VAR-GARCH model on the major and minor arterials is slightly superior to that on the expressway.

For the volatility forecasting of the volume, there are also some differences among the performance of the three models on the different roads types and speed intervals. For the expressway and major arterial, under the condition when the speed interval ranging from $30 \sim 70 \mathrm{~km} / \mathrm{h}$, the $\mathrm{KP}$ is greater than $5 \%$, and CI is large, indicating that the performance of the volatility forecasting is poor. Comparatively, when the speed is less than $30 \mathrm{~km} / \mathrm{h}$ or greater than $70 \mathrm{~km} / \mathrm{h}$, the performance is better since the $\mathrm{KP}$ is less than $5 \%$, and the $\mathrm{CI}$ is smaller. Specifically, all the models perform best when the speed is greater than $70 \mathrm{~km} / \mathrm{h}$ ( $\mathrm{KP}$ is less than $4 \%$, and $\mathrm{CI}$ is the smallest). This means that the change of traffic volume is relatively steady on the expressway and major arterial road when the speed is really low (congestion) or relatively high (free flow), and the volatility forecasting models can well capture the volatility. For the minor arterial, all the models perform well and the performance becomes better with the better traffic conditions in terms of the divided speed intervals. 
Tab. 5 Traffic volume forecasting performance comparison under different speed interval

\begin{tabular}{|c|c|c|c|c|c|c|c|c|c|c|}
\hline \multirow[b]{2}{*}{ Station } & \multirow{2}{*}{$\begin{array}{l}\text { Speed interval } \\
(\mathrm{km} / \mathrm{h})\end{array}$} & \multicolumn{3}{|c|}{ ARIMA-GARCH } & \multicolumn{3}{|c|}{ VAR-MGARCH } & \multicolumn{3}{|c|}{ ARIMA-SV } \\
\hline & & MAPE & CI & $\mathrm{KP}$ & MAPE & $\mathrm{CI}$ & $\mathrm{KP}$ & MAPE & $\mathrm{CI}$ & $\mathrm{KP}$ \\
\hline \multirow[t]{5}{*}{51008} & $<30$ & 8.68 & 78.1 & 4.60 & 8.78 & 81.0 & 3.45 & 8.68 & 73.6 & 2.30 \\
\hline & & & 7 & & & 7 & & & $\overline{2}$ & \\
\hline & $30-50$ & 8.30 & $\begin{array}{c}81.7 \\
6\end{array}$ & 6.60 & 8.60 & $\begin{array}{c}81.1 \\
7\end{array}$ & 6.32 & 8.32 & $\frac{7 \overline{5.0}}{\underline{0}}$ & 7.21 \\
\hline & $50-70$ & 8.42 & $\begin{array}{c}82.6 \\
2\end{array}$ & 7.12 & 8.55 & $\begin{array}{c}81.3 \\
9\end{array}$ & 6.80 & 8.46 & $\frac{7 \overline{7.4}}{7}$ & 7.63 \\
\hline & $>70$ & 16.98 & $\frac{59.7}{5}$ & $\underline{0.70}$ & 18.84 & $\begin{array}{c}62.7 \\
5\end{array}$ & 0.70 & 16.94 & $\begin{array}{c}40.5 \\
3\end{array}$ & 6.29 \\
\hline \multirow[t]{4}{*}{51004} & $<30$ & - & - & - & - & - & - & - & - & - \\
\hline & $30-50$ & 27.94 & $\begin{array}{c}76.5 \\
1\end{array}$ & 16.67 & 33.21 & $\begin{array}{c}62.4 \\
3\end{array}$ & 16.67 & 27.17 & $\frac{66.6}{5}$ & $\underline{8.33}$ \\
\hline & $50-70$ & 7.67 & $\begin{array}{c}78.6 \\
9\end{array}$ & 6.39 & 7.96 & $\begin{array}{c}83.2 \\
1\end{array}$ & 5.44 & 7.82 & $\frac{80.9}{3.9}$ & 4.72 \\
\hline & $>70$ & 14.99 & $\begin{array}{c}74.2 \\
1\end{array}$ & 3.73 & 14.75 & $\begin{array}{c}65.2 \\
7\end{array}$ & 4.53 & 14.59 & $\frac{56.5}{9}$ & 6.53 \\
\hline \multirow[t]{4}{*}{108121} & $<30$ & 8.84 & $\begin{array}{c}20.5 \\
2\end{array}$ & 3.26 & 10.23 & $\begin{array}{c}19.1 \\
6\end{array}$ & 4.42 & 8.89 & $\frac{20.7}{1}$ & 3.70 \\
\hline & $30-50$ & 13.95 & $\begin{array}{c}21.5 \\
5\end{array}$ & 3.66 & 15.29 & $\begin{array}{c}23.2 \\
6\end{array}$ & 5.52 & 14.06 & $\frac{20.7}{\underline{6}}$ & 3.87 \\
\hline & $50-70$ & 10.63 & $\begin{array}{c}22.7 \\
4\end{array}$ & 6.71 & 10.30 & $\begin{array}{c}21.5 \\
4\end{array}$ & 9.72 & 10.34 & $\frac{21.4}{\underline{5}}$ & 4.51 \\
\hline & $>70$ & 15.64 & $\begin{array}{c}19.9 \\
9\end{array}$ & 0.00 & 16.02 & $\frac{12.9}{\underline{5}}$ & $\underline{2.51}$ & 15.48 & $\begin{array}{c}12.7 \\
9\end{array}$ & 3.77 \\
\hline \multirow[t]{4}{*}{108221} & $<30$ & 8.93 & $\begin{array}{c}25.4 \\
5\end{array}$ & 5.22 & 7.29 & $\begin{array}{c}18.5 \\
8\end{array}$ & 7.18 & 8.88 & $\frac{24.7}{7}$ & 3.62 \\
\hline & $30-50$ & 13.57 & $\begin{array}{c}16.8 \\
8\end{array}$ & 5.03 & 13.78 & $\begin{array}{c}13.9 \\
4\end{array}$ & 9.65 & 13.85 & $\frac{15.9}{\underline{6}}$ & 3.04 \\
\hline & $50-70$ & 10.81 & $\begin{array}{c}32.6 \\
3\end{array}$ & 6.09 & 10.66 & $\begin{array}{c}31.7 \\
3\end{array}$ & 6.11 & 10.83 & $\frac{26.5}{7}$ & 3.72 \\
\hline & $>70$ & - & - & - & - & - & - & - & - & - \\
\hline \multirow[t]{4}{*}{102001} & $<30$ & 14.89 & $\begin{array}{c}29.8 \\
5\end{array}$ & 4.45 & 13.82 & $\begin{array}{c}25.4 \\
9\end{array}$ & 4.45 & 14.71 & $\frac{23.5}{6}$ & 6.53 \\
\hline & $30-50$ & 11.31 & $\begin{array}{c}27.8 \\
6\end{array}$ & 1.53 & 10.69 & $\begin{array}{c}21.0 \\
9\end{array}$ & 4.22 & 11.14 & $\frac{18.5}{7}$ & 5.37 \\
\hline & $50-70$ & 20.40 & $\begin{array}{c}27.1 \\
1\end{array}$ & 0.77 & 19.95 & $\begin{array}{c}21.8 \\
4\end{array}$ & 1.08 & 20.11 & $\frac{12.7}{1}$ & 3.84 \\
\hline & $>70$ & 32.19 & $\begin{array}{c}26.2 \\
8\end{array}$ & 0.00 & 33.23 & $\begin{array}{c}15.9 \\
8\end{array}$ & 0.00 & 32.05 & 8.28 & $\underline{0.00}$ \\
\hline \multirow[t]{4}{*}{103001} & $<30$ & 14.11 & 34.6 & 4.90 & 14.07 & 33.2 & 5.70 & 14.16 & 31.9 & $\underline{6.02}$ \\
\hline & $30-50$ & 23.69 & $\begin{array}{c}5 \\
30.2 \\
3\end{array}$ & 3.19 & 23.80 & $\begin{array}{c}4 \\
29.9 \\
7\end{array}$ & 2.26 & 23.65 & $\frac{\frac{1}{24.2}}{\underline{s}}$ & 4.52 \\
\hline & $50-70$ & 33.26 & $\frac{22.0}{3}$ & $\underline{0.00}$ & 30.29 & $\begin{array}{c}26.9 \\
8\end{array}$ & 0.00 & 32.89 & $\begin{array}{c}1 \overline{4.8} \\
0\end{array}$ & 8.00 \\
\hline & $>70$ & - & - & - & - & - & - & - & - & - \\
\hline
\end{tabular}

For the volatility forecasting of the speed, for the expressway, the performance of the models becomes better when the traffic condition is better. As seen, the KP value in the speed interval less than $50 \mathrm{~km} / \mathrm{h}$ is greater than $5 \%$, while the rest is less than $5 \%$. As for the major arterial and minor arterial, the forecasted reliability increases first and then decreases as speed increase. This indicates that the forecasted reliability enhances with the improvement of traffic conditions in the congestion dissipation period, while it decreases again in the free flow traffic state. The underlying reason may come from two aspects. On the one hand, the randomness become larger as there are a few vehicle samples in the speed interval greater than $70 \mathrm{~km} / \mathrm{h}$, and that will affect the statistical results. On the other hand, the fluctuations of traffic flow under free flow traffic condition arise normally because of the traffic interruptions by intersections, pedestrians, and non-motorized vehicles. 
Tab. 6 Traffic speed forecasting performance comparison under different speed interval

\begin{tabular}{|c|c|c|c|c|c|c|c|c|c|c|}
\hline \multirow[b]{2}{*}{ Station } & \multirow{2}{*}{$\begin{array}{c}\text { Speed } \\
\text { interval } \\
(\mathrm{km} / \mathrm{h})\end{array}$} & \multicolumn{3}{|c|}{ ARIMA-GARCH } & \multicolumn{3}{|c|}{ VAR-MGARCH } & \multicolumn{3}{|c|}{ ARIMA-SV } \\
\hline & & MAPE & CI & KP & MAPE & CI & KP & MAPE & CI & KP \\
\hline \multirow[t]{4}{*}{51008} & $<30$ & 14.56 & 26.58 & 6.90 & 12.96 & 23.48 & 6.32 & 14.18 & 23.97 & 5.75 \\
\hline & $30-50$ & 7.78 & 20.56 & 5.21 & 7.82 & 20.56 & 5.10 & 7.73 & 18.68 & 5.82 \\
\hline & $50-70$ & 5.87 & 19.24 & 2.67 & 6.01 & 19.64 & 2.73 & 5.86 & 16.88 & 4.58 \\
\hline & $>70$ & 4.80 & 18.01 & 2.80 & 4.35 & 18.35 & 1.40 & 4.72 & 16.19 & 3.50 \\
\hline \multirow[t]{4}{*}{51004} & $<30$ & - & - & - & - & - & - & - & - & - \\
\hline & $30-50$ & 24.71 & 26.17 & $\underline{50.00}$ & 23.19 & 27.13 & 50.00 & 23.40 & 41.26 & 33.33 \\
\hline & $50-70$ & 3.82 & 13.49 & 4.00 & 3.94 & 13.23 & 4.88 & 3.84 & 11.98 & 4.88 \\
\hline & $>70$ & 3.52 & 13.61 & 3.60 & 3.64 & 13.53 & 4.67 & 3.52 & 13.86 & 4.53 \\
\hline \multirow[t]{4}{*}{108121} & $<30$ & 10.22 & 9.55 & 6.63 & 10.25 & 34.20 & 0.00 & 10.07 & 9.61 & 4.42 \\
\hline & $30-50$ & 4.36 & 9.06 & 3.23 & 4.29 & 27.16 & 0.11 & 4.40 & 7.90 & 3.98 \\
\hline & $50-70$ & 3.82 & 12.46 & 4.22 & 3.79 & 12.42 & 4.34 & 3.76 & 11.69 & 4.22 \\
\hline & $>70$ & 5.22 & 13.48 & 15.48 & 5.45 & 14.96 & 12.97 & 5.17 & 13.91 & 11.72 \\
\hline \multirow{4}{*}{108221} & $<30$ & 8.47 & 9.34 & 8.55 & 8.39 & 10.78 & $\underline{6.25}$ & 8.50 & 9.07 & 9.21 \\
\hline & $30-50$ & 4.66 & 9.44 & 4.09 & 4.61 & 10.12 & 0.00 & 4.66 & 8.60 & 3.74 \\
\hline & $50-70$ & 5.92 & 14.85 & 10.97 & 5.88 & 21.94 & 4.41 & 6.03 & 13.72 & 15.67 \\
\hline & $>70$ & - & - & - & - & - & - & - & - & - \\
\hline \multirow[t]{4}{*}{102001} & $<30$ & 12.80 & 24.57 & 2.97 & 12.34 & 21.21 & 3.56 & 12.78 & 17.71 & 8.31 \\
\hline & $30-50$ & 6.52 & 19.59 & 0.48 & 6.35 & 15.35 & 0.96 & 6.51 & 11.29 & 6.33 \\
\hline & $50-70$ & 8.61 & 27.58 & 2.15 & 8.74 & 27.69 & 2.46 & 8.62 & 21.74 & 3.38 \\
\hline & $>70$ & 25.23 & 35.64 & 35.71 & 26.41 & 40.12 & 21.43 & 25.24 & 33.10 & 28.57 \\
\hline \multirow[t]{4}{*}{103001} & $<30$ & 10.31 & 14.26 & 4.02 & 10.43 & 15.24 & 2.49 & 10.34 & 12.76 & 4.90 \\
\hline & $30-50$ & 11.28 & 16.32 & 10.24 & 10.99 & 17.73 & 6.12 & 11.25 & 18.20 & 5.59 \\
\hline & $50-70$ & 29.47 & 17.27 & 64.00 & 29.23 & 20.49 & 52.00 & 29.37 & 23.16 & 56.00 \\
\hline & $>70$ & - & - & - & - & - & - & - & - & - \\
\hline
\end{tabular}

Considering the $\mathrm{CI}$ with $\mathrm{KP}$, the figures underlined in the table refer to the model with the highest forecasted reliability under the condition of the same station and same speed interval. It is found that, in most cases, the ARIMA-SV model has the best performance for both the volume and speed volatility forecasting.

\section{Conclusion}

Short-term traffic flow uncertainty forecasting is one of the key technologies in intelligent traffic operations, management and control. Considering the lack of comparative evaluation of the forecasting models under different road and traffic conditions in existing researches, the two types of short-term traffic flow parameters uncertainty forecasting models, i.e. the GARCH-type and SV models, are constructed and evaluated in this paper using the real world traffic flow data collected by the vehicle detectors. The results show that obvious difference on the model performance exists under different traffic states, but there is no model accounting for an absolute advantage. In general, the performance of the SV model on the traffic flow parameter uncertainty forecasting is slightly better than the GARCH-type models. 


\section{References}

1. Williams B, Durvasula P, Brown D. Urban freeway traffic flow prediction: application of seasonal autoregressive integrated moving average and exponential smoothing models[J]. Transportation Research Record: Journal of the Transportation Research Board, 1998 (1644): 132-141.

2. Ishak S, Al-Deek H. Performance evaluation of short-term time-series traffic prediction model[J]. Journal of Transportation Engineering, 2002, 128(6): 490-498.

3. Williams B M, Hoel L A. Modeling and forecasting vehicular traffic flow as a seasonal ARIMA process: Theoretical basis and empirical results[J]. Journal of transportation engineering, 2003, 129(6): 664-672.

4. Xie Y, Zhang Y, Ye Z. Short - Term Traffic Volume Forecasting Using Kalman Filter with Discrete Wavelet Decomposition[J]. Computer - Aided Civil and Infrastructure Engineering, 2007, 22(5): 326-334.

5. Xia J, Chen M, Huang W. A multistep corridor travel-time prediction method using presence-type vehicle detector data[J]. Journal of Intelligent Transportation Systems, 2011, 15(2): 104-113.

6. Zhang Y, Liu Y. Traffic forecasting using least squares support vector machines[J]. Transportmetrica, 2009, 5(3): 193-213.

7. Zhao-sheng Y, Yuan W, Qing G. Short-term traffic flow prediction method based on SVM [J]. Journal of Jilin University (Engineering and Technology Edition), 2006, 6: 009.

8. Davis G A, Nihan N L. Nonparametric regression and short-term freeway traffic forecasting[J]. Journal of Transportation Engineering, 1991, 117(2): 178-188.

9. Clark S. Traffic prediction using multivariate nonparametric regression[J]. Journal of transportation engineering, 2003, 129(2): 161-168.

10. Smith B L, Demetsky M J. Short-term traffic flow prediction: neural network approach[J]. Transportation Research Record, 1994 (1453).

11. Alarcon-Aquino A, Barria J A. Multiresolution FIR neural-network-based learning algorithm applied to network traffic prediction[J]. IEEE Transactions on Systems, Man, and Cybernetics, Part C: Applications and Reviews, 2006, 36(2): 208-220.

12. Zheng W, Lee D H, Shi Q. Short-term freeway traffic flow prediction: Bayesian combined neural network approach[J]. Journal of transportation engineering, 2006, 132(2): 114-121. 
13. Stathopoulos A, Karlaftis M G. A multivariate state space approach for urban traffic flow modeling and prediction[J]. Transportation Research Part C: Emerging Technologies, 2003, 11(2): 121-135.

14. Clark S. Traffic prediction using multivariate nonparametric regression[J]. Journal of transportation engineering, 2003, 129(2): 161-168.

15. Chandra S, Al-Deek H. Cross-correlation analysis and multivariate prediction of spatial time series of freeway traffic speeds[J]. Transportation Research Record: Journal of the Transportation Research Board, 2008 (2061): 64-76.

16. Chandra S R, Al-Deek H. Predictions of freeway traffic speeds and volumes using vector autoregressive models[J]. Journal of Intelligent Transportation Systems, 2009, 13(2): 53-72.

17. Nie Q, Xia J, Qian Z, et al. Use of Multisensor Data in Reliable Short-Term Travel Time Forecasting for Urban Roads: Dempster-Shafer Approach[J]. Transportation Research Record: Journal of the Transportation Research Board, 2015 (2526): 61-69.

18. Gao T. Econometric analysis methods and modeling[M]. Tsinghua University Press, 2009. (in Chinese).

19. Zhang S, Fan Z. Cointegration theory and volatility models[M]. Tsinghua University Press, 2004. (in Chinese).

20. Kamarianakis Y, Kanas A, Prastacos P. Modeling traffic volatility dynamics in an urban network[J]. Transportation Research Record: Journal of the Transportation Research Board, 2005 (1923): 18-27.

21. Guo J, Williams B, Smith B. Data collection time intervals for stochastic short-term traffic flow forecasting[J]. Transportation Research Record: Journal of the Transportation Research Board, 2008 (2024): 18-26.

22. Xia J, Nie Q, Huang W, et al. Reliable Short-Term Traffic Flow Forecasting for Urban Roads: Multivariate Generalized Autoregressive Conditional Heteroscedasticity Approach[J]. Transportation Research Record: Journal of the Transportation Research Board, 2013 (2343): 77-85.

23. Tsekeris $\mathrm{T}$, Stathopoulos A. Short-term prediction of urban traffic variability: Stochastic volatility modeling approach[J]. Journal of Transportation Engineering, 2009, 136(7): 606-613.

24. Nie Q, Xia J, Qian Z. Short-Term Traffic Flow Forecasting and Reliability Analysis of Urban Road [J]. Journal of Southwest Jiaotong University, 2013 48(5): 955-960. (in Chinese).

25. Engel R F, Kroner K F. Multivariate simultaneous generalized ARCH [J]. Econometric Theory, 1995, 11: 122-150. 
26. Tsay R S. An introduction to analysis of financial data with R[M]. John Wiley \& Sons, 2014. 